\title{
THE INCIDENCE OF DEHISCENCE OF THE TYMPANIC SEGMENT OF THE FACIAL NERVE CANAL ESTIMATED WITH COMPUTED TOMOGRAPHY
}

\section{Deniz Bulja', Dragan Stojanov², Jelena Ignjatovic3, Marija Dakovic Bjelakovic4*, Jelena Popovic5, Nebojsa Ignjatovic 6}

\author{
${ }^{1}$ Clinic of Radiology, Clinical Center of Sarajevo, Bosnia and Herzegovina \\ ${ }^{2}$ Center of Radiology, Clinical Center of Nis, Serbia \\ 3Medical Faculty, University of Nis, Serbia \\ 4Department of Anatomy, Medical Faculty, University of Nis, Serbia
}

5Department of Restorative Dentistry and Endodontics, Clinic of Dentistry, Medical Faculty, University of Nis, Serbia ${ }^{6}$ Clinic of General Surgery, Clinical Center of Nis, Serbia

\begin{abstract}
In this study, we aimed to determine the presence and incidence of dehiscence of the tympanic segment of the facial nerve canal using the High Resolution Computed Tomography (HRCT) and to evaluate this variation in relation to gender and age.

The retrospective-prospective study was performed at the Clinic for Radiology, University Clinical Center of Sarajevo. After the criteria for participation and non-participation in the study were applied, the study included 295 patients (16o women and 135 men) of ages ranging from 5 to 75, with performed computed tomography (CT) of the temporal bone as a part of a standard procedure due to the suspicion of pathological activity in that area. We divided the subjects into seven age groups. Each group included a ten-year period. The presence of dehiscence of the facial nerve canal was recorded and the obtained results were statistically analyzed.

Of the total number of patients included in the study, the dehiscence of the tympanic segment of the facial nerve canal was found in 95 patients (32\%). Of 590 temporal bones, there were 118 temporal bones with facial nerve canal dehiscence in the tympanic segment (20\%). The dehiscence without stenosis of the oval window was noted in 110 temporal bones (18.6\%). The dehiscence with stenosis of the oval window was noted in 8 temporal bones (1.4\%). There was no statistically significant difference between age groups ( $p=0.245$, Pearson's $\chi 2$ test). Also, there was no statistically significant difference between males and females ( $p=0.385$, Pearson's $\chi 2$ test).

The incidence of dehiscence of the facial nerve canal is high and the tympanic segment is the most frequent site of dehiscence localization. Almost one third of the patients with the suspicion of pathological processes on the temporal bone can have dehiscence of the tympanic segment of the facial nerve canal. HRCT is the best visual imaging technique for the evaluation of the facial nerve course within its osseous canal.
\end{abstract}

Key words: Dehiscence, facial nerve canal, tympanic segment, computed tomography

DOI: $10.21175 /$ RadProc.2016.34

\section{INTRODUCTION}

Facial expression controlled by the facial nerve represents an important means of human social communication [1], [2]. The facial nerve is longer than other nerves and its intraoseal course through the temporal bone is more tortuous. Even though the course of the facial nerve canal represents one of the most constant anatomical relationships, anomalies can occur. Anatomical variations of the facial nerve canal present a significant problem in otologic and neurootologic surgery [3]. One of the basic risks during temporal bone surgical procedures are traumatic injuries of the intratemporal segment of the facial nerve canal which may result in symptoms such as partial or complete paralysis, tics, hemifacial spasms, alterations in hearing and taste and lacrimal gland secretion.
Normally, the facial nerve canal represents a closed bony tube, apart from the sites where nerve branches exit. However, that's not always the case since the canal can be dehiscent and the nerve can herniate into the middle ear cavity [4]. Dehiscence is the most common variation of the facial nerve canal. Baxter [5] reported dehiscence in more than a half of 500 temporal bones studied microscopically. Even though the average dimension of dehiscence is smaller than $1 \mathrm{~mm}$, its existence makes the facial nerve vulnerable [6]. Dehiscence usually occurs in the tympanic segment, especially in the region of the oval window, though it can occasionally occur in the mastoid segment or near the region of the geniculate ganglion. Therefore the tympanic segment represents the most common site of iatrogenic injury of the facial nerve during otologic surgical procedure. From a surgical aspect the two most important elements in interpreting temporal

marija.bjelakovic@medfak.ni.ac.rs 
bone CT scans refer to diagnosing the pathological process and alerting surgeons to a possible anatomical variation whose presence predisposes surgical error [7]. From an anatomical aspect CT scans of bone structures enable visualization of both the temporal bone and its relationships with the surrounding topographic regions which is often impossible on anatomical specimens.

Temporal bone scanning represents an extreme challenge considering the fact that a normal anatomical structure includes a larger number of extremely small but clinically significant anatomical structures. The variety of tissues located in this region is evaluated simultaneously, making it practically impossible to develop an optimal radiological technique for the investigation of all of the potential pathological conditions. Magnet resonance imaging (MRI) has advantage in superior soft tissue resolution, whereas Multidetector Computed Tomography (MDCT) enables visualization of very delicate osseous structures, thus representing the basic method for determining developmental anomalies, traumas, acute or chronic infections or the eventual presence of pathologies within a complicated osseous structure such as the temporal bone [8]-[11]. High Resolution Computed Tomography (HRCT) performed in thin layers with a special reconstructive bone algorithm represents the best method for the facial nerve canal visualization [12]. Adequate CT scans in combination with a wellorganized protocol and adequate data from the medical history of the patient enable confirming or making an appropriate diagnosis, thus giving otorhinolaryngologists and neurosurgeons the possibility to choose the most optimal surgical approach, which is crucial for timely prevention of eventual intraoperative complications.

The aim of this study is to examine the presence and incidence of dehiscence of the tympanic segment of the facial nerve canal using HRCT temporal bone radiographies and to evaluate this variation in relation to gender and age.

\section{MATERIALS AND METHODS}

The retrospective-prospective study was carried out at the Clinic for Radiology, University Clinical Center of Sarajevo, during the period from July 2009 to December 2012. The study included 295 patients (590 temporal bones), 160 women and 135 men, ranging in age from 5 to 75 , with performed HRCT due to suspicion of pathological processes in that region. Patients with middle ear pathology, with traumatic injuries on the petrosal part of the temporal bone, as well as patients who were undergone the temporal bone surgery were excluded from the study. The patients were divided into seven groups, each group including a ten-year period. The study was approved by the Ethics committee of the University Clinical Center of Sarajevo.

Scanning was performed from the arcuate eminence of temporal bone to the mastoid tip in the transverse plane with the bone window (2300- 2500 Hounsfield units (HU) and the soft tissue window (300-500 HU). The scan image plane was parallel to the hard palate. This way, cornea was not exposed to primary X-ray beams during a CT scan. The analysis of the obtained results was performed with computed retrospective multiplanar reconstructions (MPR) which enable visualization of the scanned region in all three standard planes, and, if necessary, in additional diagonal planes. For analyzing the osseous structures of the temporal bone and all of the anatomical variations of the facial nerve canal all the scans were visualized with the window center of $700 \mathrm{HU}$ and window width of $4000 \mathrm{HU}$. Data were analyzed in computer software -GE and Siemens Syngo. Table 1 shows the used scanning protocols, depending on the used CT scanning devices.

The presence of dehiscence of the facial nerve canal was recorded and the obtained results were statistically analyzed in „IBM SPSS Statistics 20 "statistical software.

The statistical analysis included calculation of the basic parameters of descriptive statistics. Statistical significance of the mean values for each of the observed groups was tested with Pearson's $\chi^{2}$-test, and the values $\mathrm{p}<0.05$ were considered statistically significant.

Table 1. The scanning protocols, depending on the used CT scanning devices

\begin{tabular}{|l|c|c|}
\hline CT scan & $\begin{array}{c}\text { Volume Zoom, } \\
\text { Siemens }\end{array}$ & GE Lightspeed \\
\hline $\mathrm{mAs}$ & 100 & $205 \mathrm{kV}$ \\
$\mathrm{kV}$ & 120 & 120 \\
Scanning direction & Craniocaudal & Craniocaudal \\
Layer thickness & $0.5 \mathrm{~mm}$ & $0.625 \mathrm{~mm}$ \\
Collimation & $0.5 \mathrm{~mm}$ & $0.625 \mathrm{~mm}$ \\
Scanning line & Axial & Axial \\
Reconstructive & Inner Ear spi 9o & IAC \\
window & \\
\hline
\end{tabular}

\section{RESULTS}

Based on the radiological diagnostic criteria, dehiscence is defined as the absence of the osseous wall of the facial nerve canal (Figure 1). Of the total number of patients included in the study, dehiscence of the tympanic segment of the facial nerve canal was found in 95 patients (32\%). In 79 patients (26.8\%) dehiscence was found only in the tympanic segment whereas in 16 patients $(5.4 \%)$ it was observed both in the tympanic and the mastoid segment.

Of 590 temporal bones, there were 118 temporal bony with the facial nerve canal dehiscence in the tympanic segment (20\%). The dehiscence without stenosis of the oval window was noted in 110 temporal bony (18.6\%). The dehiscence with stenosis of the oval window was noted in 8 temporal bony (1.4\%). The incidence of dehiscence of the tympanic segment of the facial nerve canal in relation to gender is shown in Table 2, and in relation to age groups in Table 3 . Pearson's $\chi^{2}$-test showed that there was no statistically significant difference in the incidence of dehiscence of the tympanic segment of the facial nerve canal related to gender $(p=0.385)$. Also, there was no statistically significant difference between age groups ( $p=0.245)$. 
Table 2. The incidence of dehiscence of the tympanic segment of the facial nerve canal in relation to gender

\begin{tabular}{|c|c|c|c|}
\hline \multirow[b]{2}{*}{ Gender } & \multicolumn{3}{|c|}{$\begin{array}{l}\text { The tympanic segment } \\
\text { of the facial nerve canal }\end{array}$} \\
\hline & $\begin{array}{c}\text { No } \\
\text { dehiscence } \\
n(\%) \\
\end{array}$ & $\begin{array}{l}\text { Dehiscence of the } \\
\text { tympanic segment } \\
n(\%)\end{array}$ & $n(\%)$ \\
\hline Women & 105 (35.6) & 55 (18.6) & $(54.2)$ \\
\hline Men & $95 \quad(32.2)$ & $40(13.6)$ & $135 \quad(45.8)$ \\
\hline$\Sigma$ & $200 \quad(67.8)$ & $95(32.2)$ & 295 (100.0) \\
\hline
\end{tabular}

Table 3. The incidence of dehiscence of the tympanic segment of the facial nerve canal in relation to age groups

\begin{tabular}{|c|rr|rc|rr|}
\hline \multirow{2}{*}{$\begin{array}{c}\text { Age } \\
\text { (years) }\end{array}$} & \multicolumn{6}{|c|}{ The tympanic segment of the facial nerve } \\
& \multicolumn{2}{|c|}{$\begin{array}{c}\text { No } \\
\text { canal }\end{array}$} \\
\cline { 2 - 6 } & $\begin{array}{c}\text { Dehiscence } \\
\text { (\%) }\end{array}$ & $\begin{array}{c}\text { Dehme of the } \\
\text { tymanic segment } \\
n(\%)\end{array}$ & \multicolumn{2}{|c|}{$n(\%)$} \\
\hline $05-15$ & 23 & $(7.8)$ & 8 & $(2.7)$ & 31 & $(10.5)$ \\
$16-25$ & 16 & $(5.4)$ & 9 & $(3.1)$ & 25 & $(8.5)$ \\
$26-35$ & 20 & $(6.8)$ & 13 & $(4.4)$ & 33 & $(11.2)$ \\
$36-45$ & 39 & $(13.2)$ & 17 & $(5.8)$ & 56 & $(19.0)$ \\
$46-55$ & 41 & $(13.9)$ & 28 & $(9.5)$ & 69 & $(23.4)$ \\
$56-65$ & 52 & $(17.6)$ & 14 & $(4.7)$ & 66 & $(22.3)$ \\
$66-75$ & 9 & $(3.1)$ & 6 & $(2.0)$ & 15 & $(5.1)$ \\
\hline$\Sigma$ & 200 & $(67.8)$ & 95 & $(32.2)$ & $295(100.0)$ \\
\hline
\end{tabular}

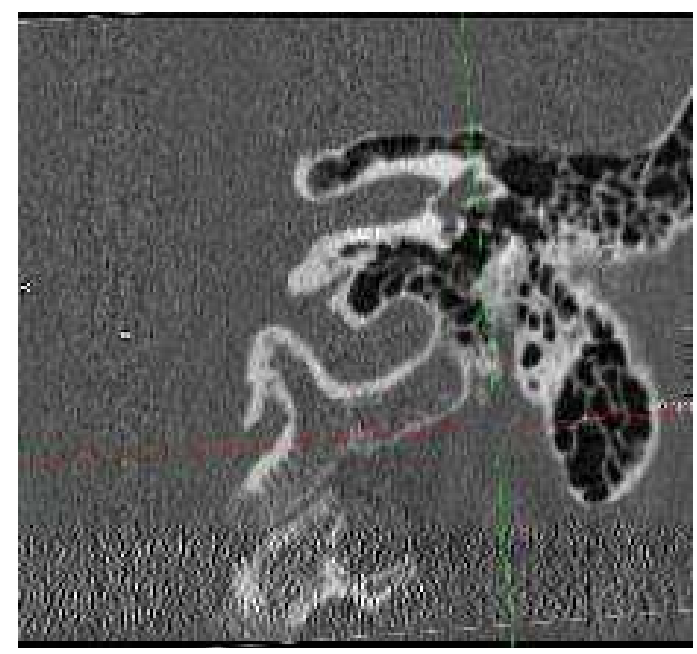

Figure 1. Dehiscence of the tympanic segment of the left facial nerve canal with the correct nerve

\section{DISCUSSION}

The facial nerve canal represents a complicated radiologic and anatomical whole. Knowing its direction and its relationship with the surrounding soft tissue structures and the eventual presence of variations are the key for success of all diagnostic and surgical procedures. The facial nerve is the most vulnerable structure in otologic surgery. Its injury during surgical procedure is not frequent but if it occurs it represents a serious complication with a very destructive effect on the patient. Since the facial nerve canal variations, especially dehiscence as the most frequent one, can represent very insidious situations for surgeons, their identification is very important for the correct planning of surgical procedures on the temporal bone. Following anatomical orientation and taking extreme caution during surgery can lead to prevention of iatrogenic injury of the facial nerve [7], [12].

Variations of the facial nerve canal have been investigated for a long period of time. The greatest number of analyses was conducted by using classical anatomical methods. The findings of the available radiologic studies seem to be uneven considering the fact that different diagnostic and radiologic criteria were used. The complete absence of the osseous wall of the facial nerve canal is usually located in the region around the oval window, in the medial $2 / 3$ of the tympanic segment. In the study of Jäger et al. [9] dehiscence was $60 \%$. According to Kim et al. [13] the incidence of dehiscence of the facial nerve canal varies from $0.5 \%$ do $74 \%$. Wang et al. [14] conducted a study on 155 temporal bones with mastoidectomy with cholesteatoma which showed incidence of dehiscence of the facial nerve canal in $29.7 \%$. Baxter [5] reported dehiscence of the facial nerve canal in $55 \%$ out of 535 temporal bones, out of which in $91 \%$ the canal was dehiscent in the tympanic segment and in $9 \%$ in the mastoid segment. Bucak et al. [3] determined intraoperatively that the incidence of dehiscence of the facial nerve canal was $28,1 \%$. The most frequent site of dehiscence $(82.8 \%)$ was the tympanic segment $(p<$ o.001). Comparing the pediatric group of patients aged under 16 with the adult group, it was reported that $10.5 \%$ had dehiscence in the pediatric group and $31.6 \%$ in the adult group. The incidence of dehiscence was more common in women but the results were not statistically significant. Nomiya et al. [15] reported the high incidence of facial nerve canal dehiscence in chronic otitis media. The incidence was $88.2 \%$ in the group of subjects aged under four years, and was $68.9 \%$ in the group of subjects aged four years and older. There was not statistically different compared to the normal controls. In our study, the incidence of dehiscence in the tympanic segment of the facial nerve canal was $20 \%$, in relation to the total number of the examined temporal bones; that is, dehiscence was observed in $32.2 \%$ of patients. There wasn't statistically significant difference in the number of dehiscence of the tympanic segment of the facial nerve canal in relation to either the gender or the age groups.

Bucak et al. [3] stated that the percentage of dehiscence of the facial nerve canal is lower in surgical than in histological studies, which can be a consequence of the osseous destruction of the facial nerve canal during the sample preparation of the cadaveric temporal bone. The other possible explanation is the presence of microdehiscence located under the facial nerve in the region of the oval window; therefore, it cannot affect microdissection that occurs intraoperatively over the lateral surface of the tympanic segment of the facial nerve canal.

Modern diagnostic techniques, such as a CT scan which presents a standard in temporal bone scanning because of its high resolution, enable visualization of quite suptile anatomical temporal bone structures. A combined analysis of axial, coronal and sagittal planes increases a positive rate in diagnosing dehiscence of the facial nerve canal. Yu et al. [16] evaluated the diagnostic value of HRCT during scanning of the tympanic segment of the facial nerve canal in patients with chronic otitis media. According to these authors, out of 76 patients with chronic suppurative otitis 
media, in $88.1 \%$ of the cases the HRCT and the surgical findings were the same. In our study, the cases with middle ear inflammation were not included in the comparative statistical analysis. We think that in such cases CT scans of all the three planes are significant only for determining the correct nerve position and not the precise determination of eventual dehiscence. However, transverse scans clearly showed the position and length of dehiscence of the lateral wall of the facial nerve. Coronal scans were better in visualizing osseous circumference of the canal and their use presents absolute imperative for determination of the absence of osseous wall of the canal especially in the area of the oval window. Sagittal layers enable better visualization of the inferior wall.

Based on the radiological and diagnostic criteria described in literature, dehiscence is defined as the absence of the osseous wall of the facial nerve canal. When the canal wall is very thin, especially in the tympanic segment where it has multiplanar and tortuous course so that it is not visible when using radiological methods of choice for the facial nerve canal analysis, the case is defined as dehiscence. For this reason some authors believe that diagnostic values of CT scans in determining dehiscence are limited [6], [14], [17], [18]. The lack of our study is the absence of comparing radiological findings with the surgical ones, considering that the patients who underwent surgery were not included in the study. However, radiological image of a substantial bone loss in any part of the facial nerve must be considered abnormal and imply dehiscence of the facial nerve canal. Because of that, a CT scan, as the best method for visualization of the osseous wall of the facial nerve canal, is necessary for documenting the presence or absence of variations in the position of the facial nerve.

\section{CONCLUSION}

The incidence of dehiscence of the facial nerve canal is high and the tympanic segment is the most frequent site of dehiscence localization. Almost one third of patients with suspicion of pathological processes on the temporal bone can have dehiscence of the tympanic segment of the facial nerve canal. HRCT is the best visual imaging technique for the evaluation of the facial nerve course within its osseous canal.

\section{REFERENCES}

1. $\quad$ R.T. Sataloff and J.B. Sataloff, Embryology \& Anomalies of the Facial Nerve and Their Surgical Implications, 2nd ed., New Delhi, India: JBMP Ltd., 2014

2. S. Yadav, A. Ranga, B.L. Sirohiwal and R. Chanda, "Surgical Anatomy of Tympanomastoid Segment of Facial Nerve," Ind. J. Otolaryngol. Head Neck Surg., vol. 58, no. 1, pp. 27-30, Jan. 2006
3. A. Bucak, S. Ulu, F. Yucedag, E. Okur, O. K. Kahveci, M. S. Tekin and A. Ayicek, "Facial Canal Dehiscence and Tympano-Mastoid Surgery," J. Int. Adv. Otol., vol. 9, no. 3, pp. 319-326, Jul. 2013

4. H.J. Gerhardt and H.D. Otto, "The Intratemporal Coruse of the Facial Nerve and its Influence on the Development of Ossicular Chain," Acta Otolaryngol., vol. 91, no. 5-6, pp. 567-573, May-June 1981

5. A. Baxter, "Dehiscence of the Fallopian Canal. An Anatomical Study," J. Laryngol. Otol., vol. 85, no. 6, pp. 587-594, June 1971

6. E. Di Martino, B. Sellhaus, J. Haensel, J.G. Schlegel, M. Westhofen and A. Prescher, "Fallopian Canal Dehiscences: A Survey of Clinical and Anatomical Findings," Eur. Arch. Otorhinolaryn. Head Neck, vol. 262, no. 2, pp. 120-126, Feb. 2005

7. W.Y. Adkins and J.D. Osguthorpe, "Management of Trauma of the Facial Nerve," Otolaryngol. Clin. North Am., vol. 24, no. 3, pp. 587-611, June 1991

8. K.S. Caldemeyer, K. Sandrasegaran, C.N. Shinaver, V.P. Mathews, R.R. Smith and K.K. Kopecky, "Temporal Bone: Comparison of Isotropic Helical CT and Conventional Direct Axial and Coronal CT," Am. J. Roentg., vol. 172, no. 6, pp. 1675-1682, June 1999

9. L. Jäger et al., "CT of the Normal Temporal Bone: Comparison of Multi- and Single-Detector Row CT," Radiology, vol. 235, no. 1, pp. 133-141, Apr. 2005

10. R. Klingebiel et al., "High-Resolution Petrous Bone Imaging Using Multi-Slice Computerized Tomography," Acta Otolaryngol., vol. 121, no. 5, pp. 632-636, July 2001

11. J. Lutz, V. Jager, M.J. Hempel, S. Srivastav, M. Reiser and L. Jager, "Delineation of Temporal Bone Anatomy: Feasibility of Low-Dose 64-Row CT in Regard to Image Quality," Eur. Radiol., vol. 17, no. 10, pp. 2638-2645, Oct. 2007

12. S. Gupta, F. Mends, M. Hagiwara, G. Fatterpekar and P.C. Roehm, "Imaging the Facial Nerve: A Contemporary Review," Radiol. Res. Pract., vol. 2013, May 2013

13. C.W. Kim, Y.S. Rho, H.-Y. Ahn and S.J. Oh, "Facial Canal Dehiscence in the Initial Operation for Chronic Otitis Media without Cholesteatoma," Aur. Nas. Lar., vol. 35, no. 3, pp. 353-356, Sep. 2008

14. H.-M. Wang et al., "Analysis of Mastoid Findings at Surgery to Treat Middle Ear Cholesteatoma," JAMA Otolaryngol. Head Neck Surg., vol. 132, no. 12, pp. 1307-1310, Dec. 2006

15. S. Nomiya et al., "Facial Nerve Canal Dehiscence in Chronic Otitis Media without Cholesteatoma," Eur. Arch. Othorinolaryngol., vol. 271, no. 3, pp. 455-458, Mar. 2014.

16. Z. Yu, Z. Wang, B. Yang, D. Han and L. Zhang, "The Value of Preoperative CT Scan of Tympanic Facial Nerve Canal in Tympanomastoid Surgery," Acta Otolaryngol., vol. 131, no. 7, pp. 774-778, Jul. 2011.

17. S.H. Selesnick and A.G. Lynn-Macrae, "The Incidence of Facial Nerve Dehiscence at Surgery for Cholesteatoma," Otol. Neurotol., vol. 22, no. 2, pp. 129-132, Mar. 2001

18. S. Yetiser, "The dehiscent facial nerve canal," Int. J. Otolaryngol., vol. 2012, Feb. 2012 Retrieved from: www.hindawi.com/journals/ijoto/2012/679708/ 